\title{
Approximately Measurement of Simplifying Tank Capacity
}

\author{
Chenghui Yang \\ College of Electrical Engineering Northwest Minzu University Gansu, China
}

\begin{abstract}
Because of the influence of displacement of oil storage tank after tank capacity table, the paper presents a new tank capacity calculation method, it has a higher accuracy and lower computational complexity, we set up a mathematical model (integral model and median model) to determine the variable parameters. Firstly, according to the hypothesis of model to analyze the problem, then according to the measured data to develop variable after the tank capacity table, finally analyze the correctness and reliability of the model. Through this method can greatly reduce the relative error, it is worth extending and applying.
\end{abstract}

Keywords-median surface model; integral models; deflection surface model; displacement identification; signing tank capacity

\section{INTRODUCTION}

In the tank capacity oil and gas's storage and transportation, it is used oval tank that both ends is spherical-capped, there are many methods to sign tank capacity, it is generally believed that liquid-sign method is the most accurate, the method includes that the quantity of liquid we measured and measuring tank in the stated height, finally making tank volume form. To the question of making tank form, it is doubt that integral is most accurate, however, integral operation is so numerous and complex. A lot of domestic and international experts put forward all kinds of approximate arithmetic, such as interpolation-algorithm, algorithmic approach and so on. It put forward a new algorithm in the passage--median surface method, on one hand, it can reduce the computational difficulty, on the other hand, it greatly improves tank capacity’s accuracy.

\section{SYSTEM DESIGN}

We can build two models, using integral model to calculate tank capacity's accuracy, then using the median model to calculate tank capacity's approximate value. We find error is approximately $0.02 \%$ after comparing, accounting for the approximate model has high accuracy.

Model hypothesis: supposing tank's shape cannot change because of external force; supposing tank's wall thickness is equal; supposing liquid in the tank leaned left; supposing the condition that ground is too soft to make slant angle too big cannot happen; supposing tank cannot excessively vertically descend. In the passage, building tank's math model, parameters used are as follows:

$\alpha$ vertically deviation angle

$H$ floater's height
$\bar{H}$ equivalent oil level

$h$ : oil height that tank body $l$ corresponded

$s(h)$ : the area of oil section in the height is $h$

$L$ : tank's length.in the title, $L=2.45 \mathrm{~m}$

$L^{\prime}$ : virtual tank body's length used in the condition of low oil level analysis

$L^{\prime \prime}$ : virtual tank body's length used in the condition of high oil level analysis

$\mathrm{V}:$ oil inflow

V': oil outflow

\section{MODEL BUILDING And ANALYSIS}

According suppose, the degree of leaning is small by too soft ground and etc. Let $\tan \alpha=b / L=1.2 / 2.45$,get $\alpha=26.0954^{\circ}$,coming from reality, there are few lean angle can exceed this value, so constructing model can be simplified. When the lean angle is smaller than $26^{\circ}$, ellipse tank level can divide into three conditions, as shown in figure1.The range below oil lavel1(low oil level), the range between oil level1 and oil level2(middle oil level), the range between oil level2 and oil level3(high oil level), because the height indicated by oil storage meter upon oil level 3 have reached the biggest value, researching the condition is meaningless, we cannot considera ${ }^{-}$it hore.

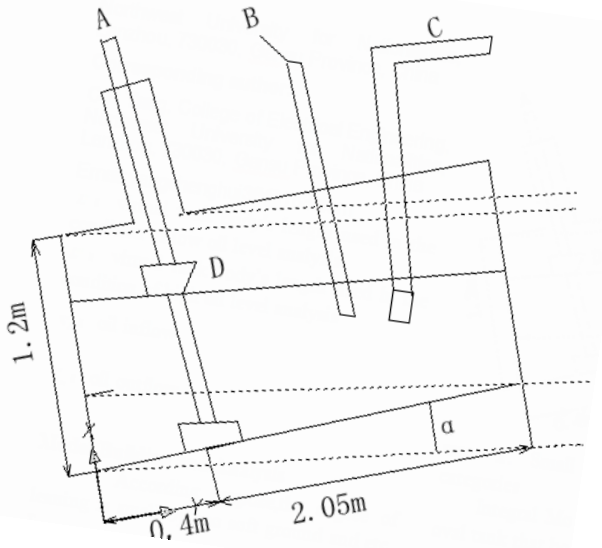

FIGURE I. SMALL ELLIPSE TANK LEVEL'S THREE CATEGORIES

Integral Model (using model1 replace it) to oval tank that both ends is even, because its shape role, so tank capacity 
function can be found using integral method.
A probe
B oil inlet
C oil out
G floater
D virtual wall
$\mathrm{N}$ horizon
P oil level

Median Surface Model (using model 2 replace it) As shown in figure 2, making symmetry plane in the center of tank, thus intersecting between the plane and oil plane is the median line. then making oil plane is perpendicular to symmetry plane. though the median line, it is the equivalent oil plane, so oil body's volume in the lean condition can transform oil body's volume in the horizontal condition.

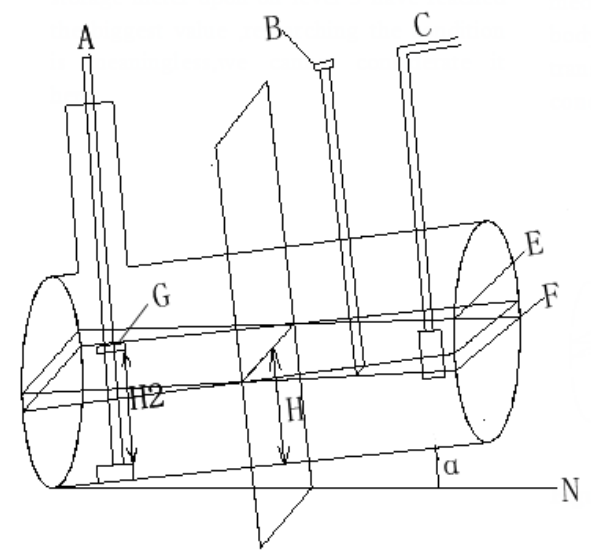

FIGURE II. ANALYSIS OF MEDIAN SURFACE MODEL

Two models need to use volume equation after displacement. Oil's volume in the oil tank can be calculated directly using floater's height. Using double integral to obtain corresponding relationship between floater's height $H$ and volume. According to simple definite integral to calculate cross section equation in the height is $h$ :

$$
\begin{aligned}
& s(h)=\frac{2 a}{b} \int_{0}^{h} \sqrt{b^{2}-(b-h)^{2}} d h \\
& =a b\left[\frac{\pi}{2}+\frac{h-b}{b} \sqrt{1-\left(\frac{h-b}{b}\right)^{2}}+\arcsin \frac{h-b}{b}\right]
\end{aligned}
$$

So, can get corresponding relationship between floater's height $H$ and volume:

$$
V_{\text {无变位 }}=s(H) * L
$$

\section{Model Piece Wise ANAlysis}

We use two models to analyze three conditions respectively

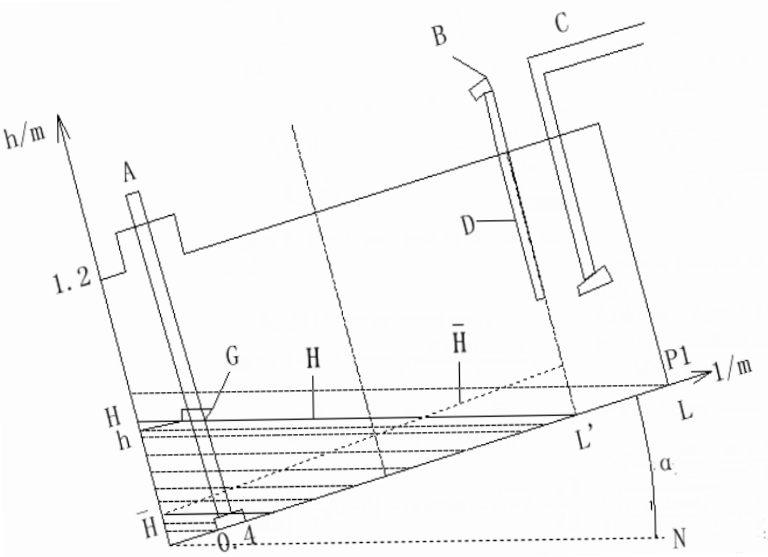

FIGURE III. SMALL OVAL TANK IN THE LOW OIL LEVEL

Model1:It can be known easily

$$
\begin{aligned}
& \text { that } h=H-(l-0.4) \tan \alpha \\
& t=\frac{H+0.4 \tan \alpha-b}{b} \text {,get } \\
& v=\int_{0}^{0.4+\frac{H}{\tan \alpha}} s(h) d l=\frac{\pi a b}{2}\left(0.4+\frac{H}{\tan \alpha}\right) \\
& +\frac{a b^{2}}{\tan \alpha}\left[-\frac{1}{3} \sqrt{\left(1-t^{2}\right)^{3}}+t \times \arcsin t+\sqrt{1-t^{2}}-\frac{\pi}{2}\right]
\end{aligned}
$$

Model 2:

the relationship between equivalent oil level $\bar{H}$ and $H$ as follow in figure 1 , get:

$$
L^{\prime}=0.4+\frac{H}{\tan \alpha} \text { and } \bar{H}=\frac{L^{\prime}}{2} \tan \alpha=\frac{H}{2}+0.2 \tan \alpha
$$

Calculating practical oil body's volume making $H$ by converting equivalent oil level, $V_{\mathrm{bw}}(H, \alpha)=V(\bar{H})$, so oil level's practical volume is:

$$
\begin{gathered}
V=s(\bar{H}) * L^{\prime}=a b L\left[\frac{\pi}{2}+\frac{\frac{H}{2}+0.2 \tan \alpha-b}{b} \sqrt{1-\left(\frac{\frac{H}{2}+0.2 \tan \alpha-b}{b}\right)^{2}}\right. \\
\left.+\arcsin \frac{\frac{H}{2}+0.2 \tan \alpha-b}{b}\right]
\end{gathered}
$$




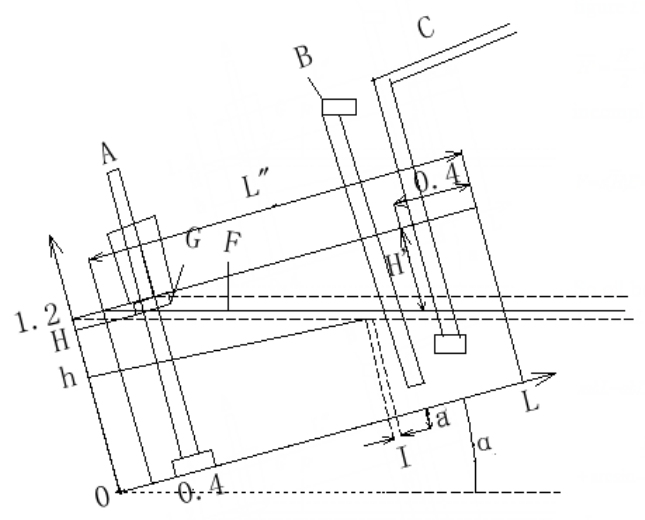

FIGURE IV. ANALYSIS OF OIL LEVEL IN MIDDLE OVAL TANK

As shown in figure4, 2.05tan $\alpha \leq H \leq 2 b-0.4 \tan \alpha$ belongs to condition of middle level

Model 1:

I $\mathrm{t}$ can be known easily $h=H-(l-0.4) \tan \alpha$, making $t=\frac{H+0.4 \tan \alpha-b}{b}$,get:

$V=\int_{0}^{L} s(h) d l=\frac{\pi a b L}{2}+\frac{a b^{2}}{\tan \alpha}\left[-\frac{1}{3} \sqrt{\left(1-t^{2}\right)^{3}}+t \times \arcsin t+\sqrt{1-t^{2}}+\right.$

$\left.\frac{1}{3} \sqrt{\left[1-\left(t-\frac{L \tan \alpha}{b}\right)^{2}\right]^{3}}-\left(t-\frac{L}{b} \tan \alpha\right) \arcsin \left(-\frac{L \tan \alpha}{b}\right)-\sqrt{1-\left(t-\frac{L \tan \alpha}{b}\right)^{2}}\right]$

Model2:

As shown in figure 4-2,get $\bar{H}=H-0.825 \tan \alpha$, so oil tank's

$$
\begin{aligned}
& \text { is } V=s(\bar{H}) * L=a b \frac{\pi}{2}+\frac{H-0.825 \tan \alpha-b}{b} \sqrt{1-\left(\frac{H-0.825 a n \alpha-b}{b}\right)^{2}} \\
& +\arcsin \frac{H-0.825 \tan \alpha-b}{b}
\end{aligned}
$$

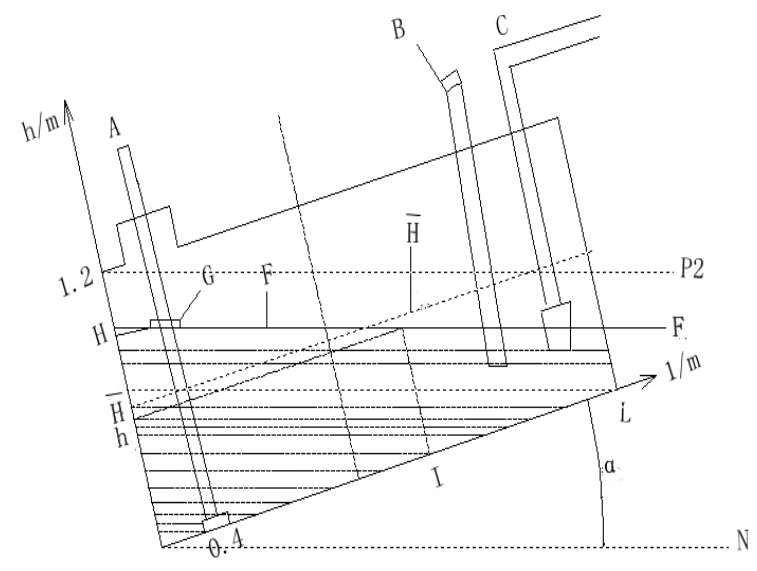

FIGURE V. SMALL OVAL TANK IN THE HIGH OIL LEVEL

As shown in figure $5,1.2-0.4 \tan \alpha \leq H \leq 1.2$ belongs to the condition of high oil level.

Model 1:

Because of left part may be submerged, and tis part is the elliptical cylinder, its height is $0.4-(1.2-H) / \tan \alpha$,so practical oil's volume is sum of the left part and the right part. $h=H-(l-0.9) \tan \alpha$,get:

$$
\begin{gathered}
V=\pi a b\left(0.4-\frac{1.2-H}{\tan \alpha}\right)+\int_{0.4 \frac{1.2-H}{\tan \alpha}}^{L} s(h) d l=\frac{\pi a b}{2}\left(0.4-\frac{1.2-H}{\tan \alpha}+L\right) \\
+\frac{a b^{2}}{\tan \alpha}\left[\frac{2}{3}+\frac{1}{3} \sqrt{\left[1-\left(t-\frac{L \tan \alpha}{b}\right)^{2}\right]^{3}}-\left(t-\frac{L \tan \alpha}{b}\right) \arcsin \left(t-\frac{L \tan \alpha}{b}\right)\right. \\
\left.-\sqrt{1-\left(t-\frac{L \tan \alpha}{b}\right)^{2}}\right]
\end{gathered}
$$

In of this, $t=\frac{H+0.4 \tan \alpha-b}{b}$

Model 2:

As shown in figure:

$$
L^{\prime \prime}=2.05+\frac{1.2-H}{\tan \alpha}
$$

and $H^{\prime}=1.2-H+1.65 \tan \alpha \quad$,so $\overline{H^{\prime}}=\frac{H^{\prime}}{2}+0.2 \tan \alpha$,thus the volume of the part incompletely filled is:

$$
V=s(\bar{H}) L^{\prime \prime}=a b L^{\prime}\left[\frac{\pi}{2}+\frac{\frac{H}{2}+0.2 \tan \alpha-b}{b} \sqrt{1-\left(\frac{\frac{H}{2}+0.2 \tan \alpha-b}{b}\right)^{2}}\right.
$$

So, oil body's volume is:

$$
V=\pi a b L-V^{\prime}=
$$


$\pi a b L-a b L^{\prime}\left[\frac{\pi}{2}+\frac{\frac{H^{\prime}}{2}+0.2 \tan \alpha-b}{b} \sqrt{1-\left(\frac{\frac{H^{\prime}}{2}+0.2 \tan \alpha-b}{b}\right)^{2}}\right.$ $+\arcsin \frac{\frac{H^{\prime}}{2}+0.2 \tan \alpha-b}{b}$

In conclusion, equation3, equation 5 , equation 7 is calculation equation used integral model after displacement of tank body; equation 4 , equation 6 , equation 8 is tank capacity equation used median surface model. Using integral model calculation to get tank capacity separated $1 \mathrm{~cm}$ each other before and after displacement, as shown in equation 3and equation 4respectively, a line of left data is calibration scale value, unit:cm; a line of right data is corresponding oil reserve in the tank, unit: L. Using median model to calculate, the error among integral models approximately is $0.02 \%$.APPENDIX 1

\section{NOTATION}

\section{$\alpha \quad$ VERTICALLY DEVIATION ANGLE \\ $H \quad$ FLOATER'S HEIGHT \\ $\bar{H} \quad$ EQUIVALENT OIL LEVEL \\ $h$ OIL HEIGHT THAT TANK BODY $l$ CORRESPONDED}

$s(h)$ THE AREA OF OIL SECTION IN THE HEIGHT IS $h$

$L \quad$ TANK'S LENGTH. IN THE TITLE, $L=2.45 \mathrm{~m}$

$L^{\prime} \quad$ VIRTUAL TANK BODY'S LENGTH USED IN THE CONDITION OF LOW OIL LEVEL ANALYSIS

L" VirTual tank BODY'S LENGTH USED IN THE CONDITION OF HIGH OIL LEVEL ANALYSIS

$\mathrm{V}$ OIL INFLOW

V’ OIL OUTFLOW
A PROBE
B OIL INLET
C OIL OUT
G FLOATER
D VIRTUAL WALL
N HORIZON
P OIL LEVEL

\section{CONCLUSION}

Through proving, calculation equation of oval tank capacity is accurate. In the calculation process, due to the influence of tank wall, it has some deviation with reality. It is worth mentioning that if considering wall's thickness, it equals to measure volume of smaller number of oil tank. If tank's thickness is $d$, so in the expression equation all

$$
\begin{aligned}
& a=a-d, \quad b=b-d \quad, \quad H=H-d \\
& L=L-2 d
\end{aligned}
$$

\section{ACKNOWLEDGMENT}

This work is supported by Fundamental Research Funds for the Central Universities (Grants 31920170079, 31920170141, \& 31920160003), by Experiment Funds of Northwest Minzu University (Grants SYSKF2017035, SYSKF2017036, SYSKF2017037, SYSKF2017043， \& SYSKF2017044), and by Program for Changjiang Scholars and Innovative Research Team in University (IRT_16R36) ( “PCSIRT16R36”).

\section{REFERENCES}

[1] A new method of oil tank calibration, OGJ (America).1992.

[2] Yun. Li, Jian-Ping. Ren, Cai-Lin Ning, The Interpolation's Application In Oil Reserves-Tech Information Development \& Economy,16(12).2006.

[3] Hong-Da. Sun, Jian-Bo. Guan, Using Approximation To Calculate Tank's Volume That Cross Section Is Spherical-capped, Fittings and Equipments, (3), 29-30.2001.

[4] Jian-Jun. Tian, Lean Horizontal Tank Straight Cylinder Section 's Volumeter, modern metrology testing.1999.

[5] Jie. Chen, MATL AB Valuable Book, Beijing, Phei.2007.

[6] LAI-Fu. LIU, Qing. He etc, using maple and matlab to solve scientific calculation problems, Beijing, CHEP.

[7] E. Q. Gao and P. Y. Feng, "Calculation of the Reserve of Horizontal Cylindrical Oil Storage Tank Declined Fitting at Different Liquid Level," Journal of Shandong Metal-lurgy, Von of the Straight Horizontal Tank,” Journ sure and Test, Vol. 5, 1999, pp. 32-36.

[8] F. J. Sun, "A Discussion on Some Difficulties in Calibran Calculation of Horizontal Oil Tank Volume,” Jour-nal of Petroleum Products Application Research, Vol. 18, No. 5, 2000, pp. 20-24.

[9] X. G. Pan, "Measurement and Calculation of Tilt Hori-zontal Tank Volume,” Journal of Oil \& Gas Storage and Transportation, Vol. 6, No. 6 , 1987, pp. 47-50.

[10] http://www.mcm.edu.cn/html_cn/node/d5ae730f57dea3208cae73f7635a eee8.html.

[11] Code for design of vertical cylindrical welded steel oil tanks. GB 50341-2003.2003.12.1

[12] Specification for field welded tanks for storage of production liquids. SY/T 0606-2006 